\title{
Endoscopic Management of Optic Nerve Sheath Meningocele
}

\author{
${ }^{1}$ Ravi Meher, ${ }^{2}$ Anoop Raj, ${ }^{3}$ Pankaj Vats, ${ }^{4}$ Deepti Vats, ${ }^{5}$ Subash Dadeya \\ ${ }^{1}$ Assistant Professor, Department of ENT, Maulana Azad Medical College and Associated Lok Nayak Hospital, New Delhi, India \\ ${ }^{2}$ Professor and Head, Department of ENT, Maulana Azad Medical College and Associated Lok Nayak Hospital, New Delhi \\ India \\ ${ }^{3}$ Ex Senior Resident, Ophthalmology, Maulana Azad Medical College and Associated GNEC, New Delhi, India \\ ${ }^{4}$ Ex Resident, Ophthalmology, Maulana Azad Medical College and Associated GNEC, New Delhi, India \\ ${ }^{5}$ Associate Professor, Ophthalmology, Maulana Azad Medical College and Associated GNEC, New Delhi, India
}

Correspondence: Ravi Meher, Assistant Professor, Department of ENT, Maulana Azad Medical College and Associated Lok Nayak Hospital, New Delhi, India, e-mail: ravimeher@gmail.com

\begin{abstract}
Optic nerve sheath dilatation forming a cyst containing cerebrospinal fliud (CSF) is a rare condition. It is actually an enlargement and dilation of primarily the optic nerve sheath. There is an expansion of the cerebrospinal fliud space around the optic nerve with no associated inflammation, orbital or cerebral neoplasm at the apex of the orbit. It is a very rare condition and only few cases have been reported in the literature. We describe a case of optic nerve meningocele which was successfully managed surgically through intranasal route. A brief review of the existing literature is also presented.
\end{abstract}

Keywords: optic nerve meningocele, optic nerve cyst, CSF.

\section{INTRODUCTION}

The optic nerve in its intracanalicular and intraorbital course is covered with all three meningeal layers while in intracranial part it is only sheathed with the pia mater, making it behave as an out pouching of brain. This may sometimes lead to formation of CSF containing cyst occurring in the orbital region.

\section{CASE REPORT}

A 32 years old male patient presented to out patient department with complaints of gradually increasing painless proptosis of left eye over past 6 months. The patient had no other significant ocular or systemic complaints. On examination the proptosis was lateral, nonpulsatile, nonintermittent. On palpation there was no definite swelling. Orbital margins could be palpated easily. Hertel's exophthalmometry showed reading of $18 \mathrm{~mm}$ in right eye and $26 \mathrm{~mm}$ in the left eye with bar reading of $110 \mathrm{~mm}$. No mass or bony defect was palpable in any quadrant. The visual acuity was $6 / 6$ both eyes. Rest of the anterior and posterior segment examination was normal. Over a period of next 4 months, the proptosis increased with increase in intraocular pressure and the visual acuity dropped to $6 / 18$ in affected eye even while the patient was on Acetazolamide. The right eye was normal. Blood profile and radiological investigations were done to exclude systemic illness and assess ocular problem. Thyroid function test was normal. $\mathrm{X}$-ray orbit antero-posterior and lateral view showed no bony defect's. CT scan orbit showed a hypodense cystic lesion medial compartment of left abutting the optic nerve (Fig. 1). A clinical correlation of investigations led to suspicion of CSF cyst arising from optic nerve meninges. Two surgical approaches, i.e. external medial approach and endonasal, which were feasible, were assessed. The patient was planned for endoscopic endonasal cyst aspiration. After uncinectomy and anterior ethmoidectomy the lamina papyracea and orbital fat were removed to reach the medial compartment of the orbit. The cyst was then identified and completely aspirated and sent for chemical analysis which confirmed it to be CSF. Patient improved dramatically with proptosis disappearing immediately (Fig. 2) and visual acuity improved to 6/6 in both eyes. Patient is on regular followup for 6 months and is asymptomatic.

\section{DISCUSSION}

The term optic sheath meningocele has been proposed by Garrity and Forbes ${ }^{1}$ to describe primary CSF cysts of the 

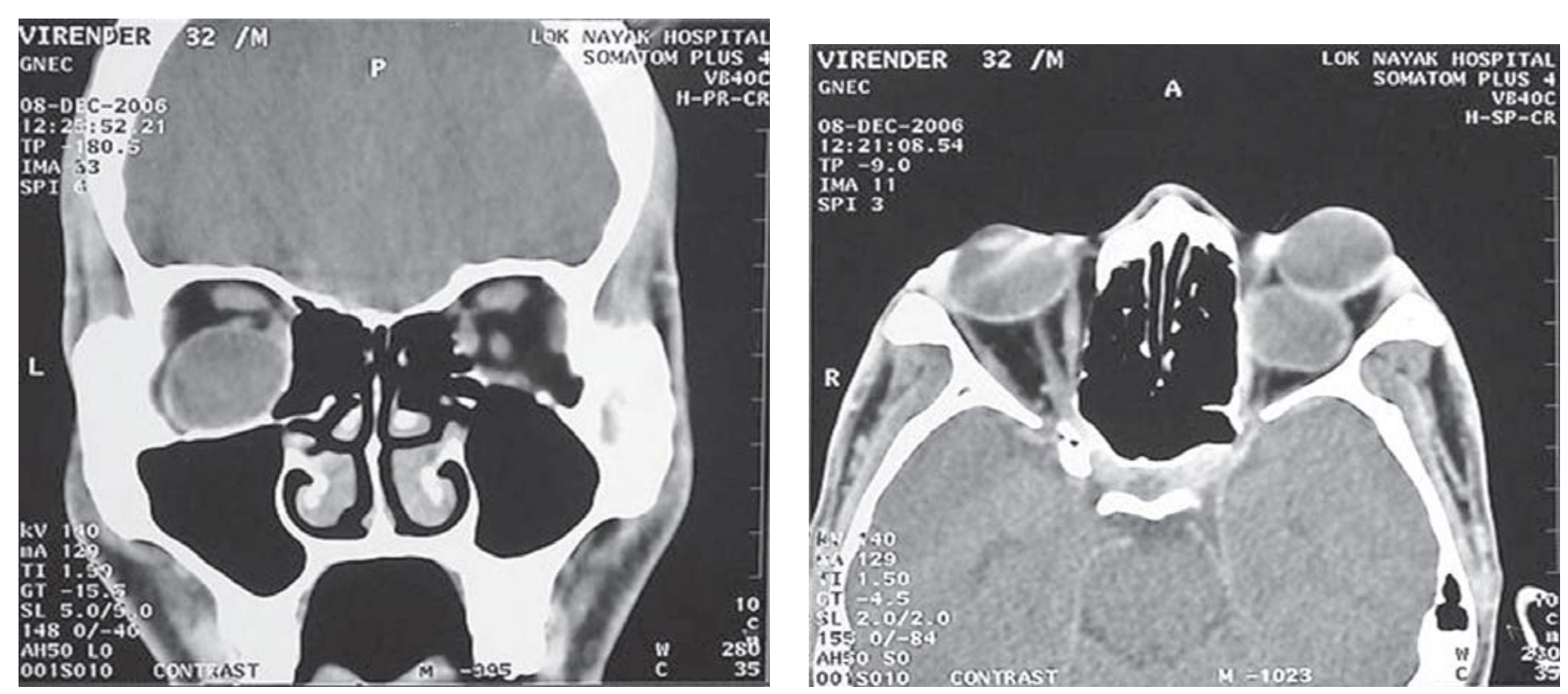

Figure 1: CT scan axial and coronal cuts showing a hypodense cystic lesion in medial compartment of left orbit abutting the optic nerve
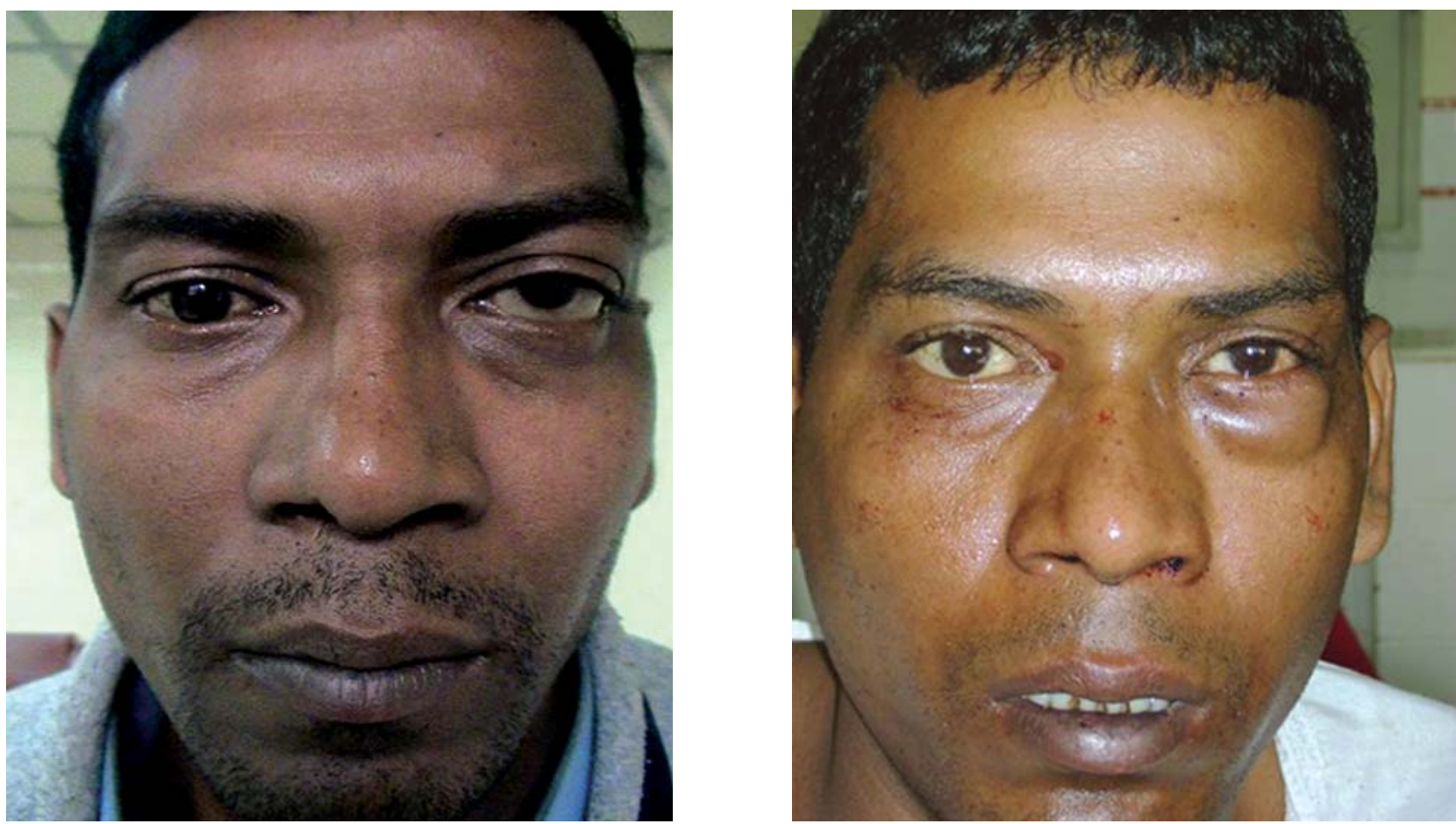

Figure 2: Clinical photograph of the patient: Preoperative and immediate postoperative

optic nerve sheath, without apical mass or malformation of the cranio-orbital junction. The terms arachnoid cysts, optic hydrops ${ }^{2}$ and patulous subarachnoid space have been used to describe this clinical entity. To date there have been over 32 cases described in the literature., ${ }^{3,4}$ On reviewing the literature two subsets of patients with idiopathic optic sheath meningocele have been reported. The first is a group in which there is concurrent evidence of progressive hyperopia and choroidal folds. This typically affects middle-aged males. It is possible to have this as an isolated entity without evidence of an associated meningocele. In a case series by Dailey et al four out of the seven patients who reported with hyperopia and choroidal folds had optic nerve meningoceles. ${ }^{5}$ Several of the patients had associated 
changes at the macula which included a retinal pigment epitheliopathy and central serous retinopathy. The role of raised intracranial pressure is unclear in the etiology as only a few of the reported cases have had raised intracranial pressure.

The second subgroup includes optic nerve meningoceles with no underlying pathology. This group has excellent prognosis once the cyst is treated surgically. Our patient belonged to second group.

Presenting symptoms are often related to involvement of the optic nerve, with a slow or rapid decrease of visual acuity; rise in intraocular pressure, headache, retro-orbital pain, proptosis and in some cases cystoid macular edema. ${ }^{6}$ It may be unilateral or bilateral. In our patient there was involvement of only one eye with proptosis and decreased visual acuity. CT and MRI studies reveal a tubular-cystic enlargement of the optic nerve, optic sheath complex, with thickening of the optic nerve. Radiological differential diagnosis includes optic nerve tumors such as gliomas, meningiomas, and arachnoid cysts involving the optic nerve sheath. ${ }^{7}$ Mesa-Gutiérrez et al reports a patient with optic nerve sheath meningocele treated with Acetazolamide who showed a maintained improvement during a 2-year followup period. ${ }^{8}$ In cases where there is no improvement on medical treatment, surgical decompression is the treatment of choice. Early surgical management by means of optic nerve/optic nerve sheath decompression affords an improvement of visual function with minimal morbidity in patients who present a rapid decrease of visual acuity within 3-6 months. Patients in both groups can develop progressive visual loss. In these cases performing early surgical decompression of the optic nerve sheath should be considered. This may improve vision or halt the progression in some cases. However it is believed that all cases with visual loss should have a thorough examination of the macula and electrophysiological testing to determine the contribution of optic nerve pathology to visual loss before surgery is contemplated.

\section{REFERENCES}

1. Garrity JA, Trautmann JC, Bartley GB, Forbes G, Bullock JD, et al. Optic nerve sheath meningoceles. Clinical and radiographic features in 13 cases with a review of the literature. Ophthalmology 1990;97:1519-31.

2. Jinkins JR. Optic hydrops: Isolated nerve sheath dilation demonstrated by CT. AJNR Am J Neuroradiol Sep-Oct 1987;8(5):867-70.

3. Jungkim S, Khurshid SG, Fenton S. Dural ectasia of the optic nerve sheath. Acta Ophthalmol Scand Apr 2005;83(2):266-67.

4. Lunardi P, Farah JO, Ruggeri A, Nardacci B, Ferrante L, et al. Surgically verified case of optic sheath nerve meningocele: Case report with review of the literature. Neurosurg Rev 1997;20:20105 .

5. Dailey RA, Mills RP, Sticmac GK, et al. The natural history and CT appearance of acquired hyperopia with choroidal folds. Ophthalmology Oct 1986;93(10):1336-42.

6. Shanmuganathan V, Leatherbarrow B, Ansons A, Laitt R. Bilateral idopathic optic nerve sheath meningocele associated with unilateral transient cystoid macular edema. Eye 2002;16:80002 .

7. Imes RK, Hoyt WF, Brodsky MC. Meningocele or gliomatosis? Ophthalmology May 1991;98(5):562.

8. Mesa-Gutiérrez JC, Quiñones SM, Ginebreda JA. Optic nerve sheath meningocele. Clin Ophthalmol Sep 2008;2(3):661-68. 